\title{
Evolutionary synthesis models for the formation of S0 galaxies in clusters
}

\author{
J. Bicker, U. Fritze-v. Alvensleben, and K. J. Fricke \\ Universitätssternwarte Göttingen, Geismarlandstr. 11, 37083 Götingen, Germany
}

Received 17 December 2001 / Accepted 18 March 2002

\begin{abstract}
Rich galaxy clusters in the local Universe show a large population of S0 galaxies ( $40 \%$ of all luminous galaxies). With increasing redshift the fraction of this S0 galaxy population is observed to strongly decrease (e.g. by a factor $\sim 2-3$ to $z=0.5$ ) in favor of the spiral galaxy fraction while the number of bright ellipticals does not seem to change. The infalling field galaxy population that successively builds up the cluster also is spiral rich and S0 poor. It has hence been suspected that galaxy transformation processes, either due to galaxy - galaxy or to galaxy - ICM interactions, are responsible for this change. Complementing dynamical and morphological studies, we use evolutionary synthesis models describing various possible effects of those interactions on the star formation rates of the infalling spirals. We study the effects of starbursts of various strengths as well as of the truncation of star formation on the color and luminosity evolution of model galaxies of various spectral types. Comparison with observed properties of the local S0 galaxy population is used to constrain possible S0 formation mechanisms. We find that star formation truncation in spiral galaxies earlier than Sd-type, if occurring not too long ago, as well as starbursts more than 3 Gyr ago and followed by complete star formation extinction in spirals - again earlier than Sd- may well account for the observed average S0 luminosities and colors. Late-type galaxies (Sd), even after a strong burst, remain either too blue or too faint. Our results are in agreement with studies of spectral features of cluster S0s but allow for stronger constraints.
\end{abstract}

Key words. galaxies: formation, evolution, interactions, starburst, elliptical and lenticular, cD - galaxies: clusters: general

\section{Introduction}

The galaxy populations in rich clusters are strikingly different at intermediate and high redshift from what they are in the local Universe. As early as 1978 Butcher \& Oemler noticed a large fraction of blue galaxies in the centers of distant clusters in contrast to the situation in the centers of nearby rich galaxy clusters where almost exclusively red and passive early-type galaxies are found. While the early observations referred to color or spectral classification of galaxies, HST has opened up the possibility for direct morphological classifications of distant galaxies.

While the composition of the bright field galaxy population in terms of morphological types (E, S0 and Spirals), does not seem to change significantly up to $z \geq 0.5$, that of the populations in rich galaxy clusters does. S0 galaxies, in particular, constitute $\sim 40 \%$ of the local cluster population while 10 rich clusters at $z \sim 0.5$ have been shown to only contain $\sim 15 \%$ S0 galaxies (Dressler 1980; Dressler \& Gunn 1992; Dressler et al. 1997, 1999; Smail et al. 1997). This trend has been confirmed by additional studies of

Send offprint requests to: J. Bicker, e-mail: jbicker@uni-sw.gwdg.de
12 clusters in the redshift range between $z \sim 0.1$ and 0.3 by Couch et al. (1996) and Fasano et al. (2000). The proportion of elliptical galaxies in the central regions of rich clusters does not seem to change significantly with redshift while that of the spirals does in a sense opposite to that of the S0s.

Structure formation scenarios show that even in the local Universe galaxy clusters are still in their process of formation by continuously accreting galaxies from the spiral-rich field environment.

Various processes have been discussed that might act to transform infalling star-forming field spirals into the passive S0 galaxy population seen inside nearby rich clusters like e.g. Coma. This transformation has two aspects: the morphological transformation and the truncation of the star formation rate (SFR). The timescales for both processes may or may not be different.

Spiral-spiral mergers are known from dynamical \& spectrophotometric modeling and from the multi-frequently observations of nearby examples (NGC 4038/39, NGC 7252) to lead to spheroidal remnants of type S0/E, excite sometimes very strong bursts of SF during the interaction, consuming much of the gas, 
eventually expelling the rest, leaving a gas-poor remnant with low or vanishing SFR and colors \& spectral features evolving from those of a typical postburst galaxy with strong Balmer absorption lines to those of "normal" E/S0 galaxies (Toomre \& Toomre 1972; Barnes \& Hernquist 1996; Fritze-v. Alvensleben \& Gerhard 1994; Schweizer 1999).

The high velocity dispersions of galaxies in rich clusters today, however, are unfavorable to galaxy merging. During the early stages of cluster formation or within galaxy groups falling into a cluster, However, merging may play an important role. Harassment has been shown by Moore et al. $(1996,1998)$ to be efficient in stripping stars from spiral disks during their high speed encounters with other cluster members and/or inhomogeneities in the cluster potential. However, only for massive bulge-dominated infalling galaxies S0-type remnants are expected. Latetype or low surface brightness galaxies will rather be shred down to dSphs or dEs.

Beyond these galaxy-galaxy and galaxy-potential interactions a third type of interaction is shown to be important, both observationally and from theoretically modeling: that of a galaxy with the dense hot ICM filling the central regions of clusters - at least in the local Universe as seen in X-ray observations. As this hot ICM can reach densities up to a factor 100 higher than those of the ISM in spiral galaxies it will cause strong shocks in the ISM of spiral galaxies falling towards the cluster center as well as efficient ram pressure stripping or sweeping of its ISM (Gunn \& Gott 1972; Abadi et al. 1999; Fujita 2001). Quilis et al. 2000 present 3D hydrodynamic simulations with good shock resolution showing that ram pressure together with turbulent and viscous stripping can remove a galaxy's HI content and hence extinguish its SF within $\sim 10^{8} \mathrm{yr}$. Preexisting molecular clouds and the strong shocks induced within the ISM may give a rise to starburst during this process.

Both hydrodynamic models and observations, showing that all of the starburst and most of the post-burst galaxies in clusters are spirals, indicate that changes in SFR take place on shorter timescales $\left(\leq 10^{8} \mathrm{yr}\right)$ than morphological transformations $\left(\sim 10^{9} \mathrm{yr}\right)$.

Observationally, the hydrogen deficiency of spiral galaxies near or around the central regions of dynamically relaxed clusters and their asymmetric or displaced HI distributions are direct evidence of the effect of the hot ICM (Cayatte et al. 1990; Bravo-Alfaro et al. 2000; Poggianti \& van Gorkom 2001). The spectacular $\mathrm{H}_{\alpha}$ emission of some Virgo and A1367 galaxies as they are stripped confirms the starbursts predicted to be induced during this process (Gavazzi et al. 1995; Kenney \& Koopman 1999).

A delay of 1-2 Gyr between SF truncation and the morphological transformation in a infalling spiral is consistent with the lack of blue S0s in distant clusters as well as with the large number of passive galaxies with latetype morphologies (Poggianti et al. 1999; Kodama \& Smail 2001; Smail et al. 2001). Ziegler (2000) and Smail et al. (2001) find that while luminous E/S0s show optical-NIR colors and spectral line strengths consistent with old systems and passive evolution since $z>0.5$, the fainter E/S0s $\left(\leq 0.1 L_{K}^{*}\right)$ show a large spread in optical and optical-NIR colors with $\sim 30 \%$ of the S0s in A2218 $(z=0.17)$ having luminosity-weighted ages $\leq 5 \mathrm{Gyr}$, i.e. they were actively forming stars at $z \leq 0.5$. This proportion of young S0s is consistent with the rate of evolution in the fraction of S0s in clusters over the redshift range from $z \sim 0.5$ to $z=0$ (Dressler et al. 1997). Similar young components are reported for 3 other S0 galaxies at the periphery of the Fornax cluster by Kuntschner (2000).

While most of these studies have concentrated on the inner regions of rich clusters, both at high and low redshift, it is clear that, in fact, the evolution of galaxy clusters themselves, in terms of richness, degree of relaxation, and content of ICM have to be included into any analysis of the evolution of their galaxy populations. Very first attempts of this kind are presented by Balogh et al. (2000).

In this paper we take a much more modest approach. We use evolutionary synthesis models that were earlier proven to correctly describe the evolution of undisturbed field galaxies of various spectral types over significant look-back times and we artificially modify their SFRs at different evolutionary stages as suggested to happen when these galaxies are assumed to fall into a cluster. I.e. we induce a burst of SF and/or truncate the SF on timescales as indicated by observations and/or hydrodynamical simulations. We then investigate the subsequent evolution of our model galaxies in terms of optical and optical-NIR colors and luminosities and compare with the corresponding data for the local S0 cluster galaxy population to try and constrain possible photometric evolutionary paths from field spirals to cluster S0s. It may be of historical interest that already in 1975 Biermann \& Tinsley presented an early version of an evolutionary synthesis study, conducted mainly in Göttingen, on the effect of SF truncation in spirals in comparison with cluster S0s, on the basis of stellar input physics (main sequence evolutionary tracks for solar metallicity stars) and computing facilities available at that time.

We present the basic principles of our models in Sect. 2, discuss the results from a grid of models for different spiral types experiencing different changes of their SFR at various evolutionary stages in Sect. 3, and compare to observational properties of local S0 galaxies in Sect. 4. Section 5 summarizes our conclusions, compares to results from other groups studying spectral features, and gives an outlook to future developments of our models an observations.

\section{The spectrophotometric evolutionary model}

\subsection{Undisturbed galaxies}

The evolutionary synthesis model we use is based on Tinsley's equations, which describe the time evolution of the gas mass, the stellar mass, and the ISM metallicity on the basis of a given initial mass function (IMF) and a 
star formation rate in its time evolution specific for each galaxy type (e.g. Tinsley 1980). At the same time our code follows the detailed evolution of the stellar population using a complete set of stellar evolutionary tracks including all relevant stellar evolutionary phases, as given by the Geneva group (cf. Weilbacher et al. 2000 for details), stellar yields and lifetimes. It is a 1-zone model for the description of global galaxy properties. Starting from a homogeneous gas cloud of initial mass and chemical composition our program calculates the distribution of the stellar population over the HRD at every timestep. The model assumes ideal gas mixing and no in- or outflow of gas, but accounts for the finite lifetimes of stars of different masses. The various spiral galaxy types are described by conventional global star formation rates. For $\mathrm{Sa}, \mathrm{Sb}$, and Sc galaxies we assume SFRs which are linear functions of the evolving gas content $G(t), \psi(t) \sim G(t)$ (Schmidt 1959; Kennicutt 1998). The Sd model has a constant SFR. These star formation rates are close to those empirically determined by Sandage (1986), and also used by Bruzual \& Charlot (1993) and Guiderdoni \& RoccaVolmerange (1987). The IMF is taken from Scalo (1986):

$\phi(m) \mathrm{d} m \propto m^{-(1+x)} \mathrm{d} m$

with lower and upper mass limits $m_{1}=0.15 M_{\odot}$ and $m_{\mathrm{u}}=85 M_{\odot}$. The three different slopes are $x_{1}=0.25$ for $m_{1} \leq m<1, x_{2}=1.35$ for $1 \leq m<2$ and $x_{3}=2.00$ for $2 \leq m \leq m_{\mathrm{u}}$. To account for dark matter in the form of brown dwarfs or planets the normalization

$\int_{m_{\mathrm{l}}}^{m_{\mathrm{u}}} \phi(m) m \mathrm{~d} m=0.5$

(Bahcall et al. 1992) is used which, after a Hubble time, gives mass-to-light ratios for our model galaxies in agreement with observations. We use the tracks for a metallicity of $\frac{1}{2} Z_{\odot}$, which give a good description for stars in spiral galaxies (Rocha-Pinto \& Maciel 1998).

The model calculates the time evolution of luminosities in the Johnson $U B V R I J H K$ bands and the corresponding colors.

The SFRs chosen above were carefully checked to yield agreement after $12 \mathrm{Gyr}$ - the present galaxy age in our cosmology - with average observed integrated colors from $U$ through $K$ for the respective galaxy types (RC3, Buta et al. 1994) At an age of 12 Gyr (today), the total $B$-band luminosities of the different undisturbed galaxy models are scaled to the average $\left\langle M_{B}\right\rangle$ from Gauss-fits to observed type-specific luminosity functions of the respective galaxy types in Virgo as derived by Sandage et al. (1985). This scaling also is applied to the total, stellar and gaseous masses of the model galaxies.

\subsection{Interacting galaxies}

To model interacting galaxies we use two different scenarios. Starbursts are used to describe merging galaxies or galaxies with high SFRs triggered by shock waves in the ISM of a galaxy falling into the hot dense ICM. Star formation truncation, on the other hand, is used to model galaxies which lose their gas by tidal interaction or by ram pressure stripping when falling into a cluster.

In case of the truncation we simply stop star formation at a given point in time. A burst is described by a sudden increase of the SFR followed by an exponential decline either to zero or to some given value $\psi_{\mathrm{f}}$ assumed to be constant thereafter.

$-\psi_{\max }: \max . \mathrm{SFR}$ at the beginning of the burst, i.e. at $t_{\text {burst }}$;

- $\tau_{\text {burst }}$ : exponential decline timescale of the burst SFR, typically $\sim 10^{8} \mathrm{yr}$

$-\psi_{\mathrm{f}}=0$ or $=1.5 M_{\odot} \mathrm{yr}^{-1}$ : eventual low level constant SFR after the burst.

The strength of the burst $b$ is defined by the fraction of gas available at the time when the burst starts $G\left(t_{\text {burst }}\right)$, which is consumed for star formation during the burst $(\Delta G)$ :

$b:=\frac{\Delta G}{G\left(t_{\text {burst }}\right)}$.

Another common definition of the burst strength is the fraction of stars built in the burst $(\Delta S)$ as compared to the total stellar mass of the galaxy by today $\left(S\left(t_{0}\right)\right)$ :

$b_{*}:=\frac{\Delta S}{S\left(t_{0}\right)}$.

\section{Model results}

With the code described above we compute a grid of models for spiral galaxies falling into a cluster and having their SFRs affected by starbursts and/or star formation truncation as described above. The different parameters are:

- Spectral types Sa, Sb, Sc, and Sd of the infalling galaxies - defined by their respective SF histories;

- evolutionary age of the galaxies at the onset of the burst or the star formation truncation: 3, 6, and 9 Gyr

and in case of a starburst

- burst strength b in terms of gas consumption: $70 \%$, $50 \%$, and $30 \%$ (strong, intermediate and weak bursts);

- SFR after the burst $\psi_{\mathrm{f}}=1.5 M_{\odot} \mathrm{yr}^{-1}$ or $\psi_{\mathrm{f}}=0$.

In Fig. 1 the time evolution of the B-luminosity for an Sc galaxy with different interactions affecting its SFR after 6 Gyr of undisturbed evolution is shown. The flat curve shows the evolution of an undisturbed Sc galaxy. As a result of its star formation rate decreasing slowly as the gas content decreases, the $B$-band luminosity of the undisturbed Sc galaxy model (dot-dashed) also decreases very little after its maximum is reached at $\sim 2$ Gyr of evolution. After 12 Gyr of undisturbed evolution the average $\left\langle M_{B}\right\rangle \sim-18.3 \mathrm{mag}$ of Virgo Sc galaxies is reached. If the star formation is truncated at 6 Gyr as shown by the 


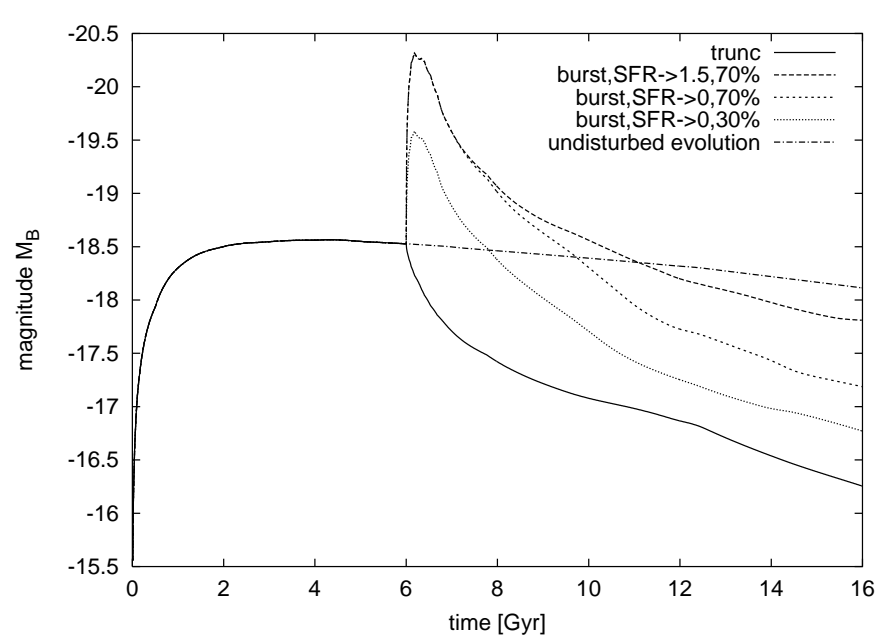

Fig. 1. Time evolution of $B$-band luminosities of Sc galaxies in different interaction scenarios with the SFR affected by strong and weak bursts and SF truncation after 6 Gyr of undisturbed evolution.

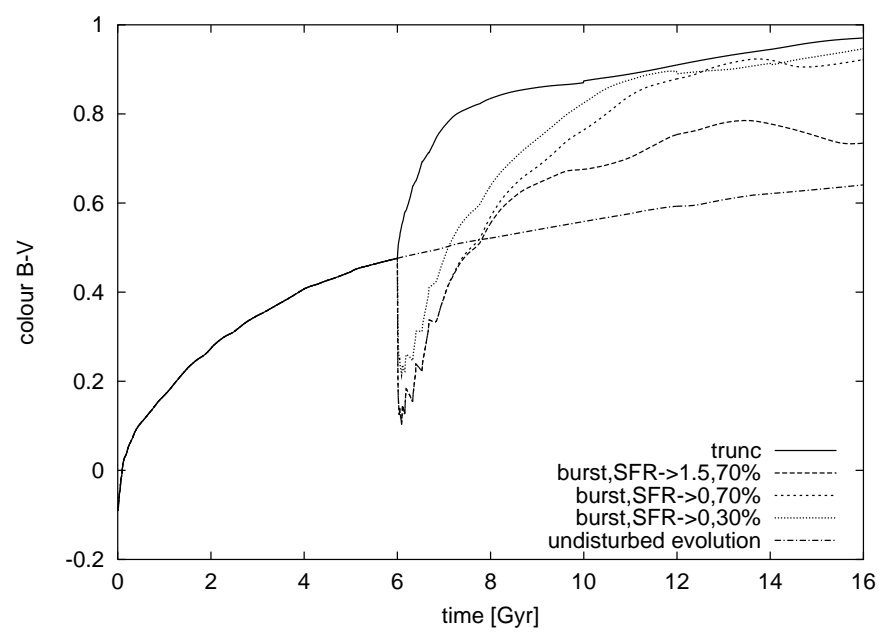

Fig. 2. Time evolution of the $B-V$ color for the same models as in Fig. 1

solid curve, the $B$-luminosity decreases appreciably and only amounts to $M_{B}=-16.9 \mathrm{mag}$ at an age of $12 \mathrm{Gyr}$. Bursts of various strengths, consuming 30 and $70 \%$ of the remaining gas reservoir, lead to a strong and rapid luminosity increase to $M_{B}=-19.5$ and $M_{B}=-20.3 \mathrm{mag}$ for the weak and strong burst cases (dotted and dashed lines), respectively. About $5 \times 10^{8}$ yr after the onset of the burst, systems start fading again and 2 Gyr (4 Gyr) after the weak (strong) burst, the post-burst galaxy becomes fainter than the undisturbed one. If some residual star formation is assumed at a constant rate of $1.5 M_{\odot} \mathrm{yr}^{-1}$ after the burst (long dashed line), the fading gets weaker.

Figure 2 shows the color evolution in $B-V$ for the same models as in Fig. 1. The flat dot-dashed curve is the undisturbed Sc galaxy again. It describes the successive reddening of its aging stellar population. With the star formation rate we assumed, the Sc model, after 12 Gyr of undisturbed evolution, indeed reaches the average $B-V=0.59$ of Sc galaxies in the RC3 (Buta et al. 1994). If star formation is truncated after 6 Gyr of evolution (solid line) a strong and very sudden reddening results due to the massive stars dying out very fast. 2 Gyr after star formation truncation, $B-V=0.84$ is reached, whereafter the further reddening is much slower and reaches $B-V=0.91$ at 12 Gyr. If a burst occurs after $6 \mathrm{Gyr}$ of evolution, colors rapidly get very blue, the difference between a strong burst $(B-V=0.1$, dashed line) and a weak one $(B-V=0.2$, dotted line) not being large. After the burst, $B-V$ reddens again, though more slowly than in the truncation case. The $B-V$ of the undisturbed model is reached $1.5 \mathrm{Gyr}$ after a weak and 1.8 Gyr after the strong burst. Thereafter the reddening continues until, at an age around $12 \mathrm{Gyr}, B-V$ is almost as red as in the truncation model. If star formation does not go to zero after the burst but continues at some low constant level, $B-V$ stays significantly bluer $(B-V) \sim 0.75$. Note that while burst models with $\psi_{\mathrm{f}}=0$ and star formation truncation models cannot be distinguished in $B-V$ any more at times $\geq 5$ Gyr after the event, they are still very well distinguished in terms of luminosity.

Here, as an example, we only present the $B$-luminosity and $B-V$-color evolution. Our models, however, comprise all optical and NIR luminosities and colors from $U$ through $K$ and they all behave in their specific ways in burst and truncation models.

For undisturbed galaxies of all types $\mathrm{Sa}, \mathrm{Sb}, \mathrm{Sc}$ and $\mathrm{Sd}$, of course, star formation rates were chosen as to provide agreement of all colors from $U-B$ through $V-K$ at an evolutionary age of $\sim 12$ Gyr with their respective average values as observed for nearby galaxy samples.

Because of their initially higher and more rapidly decreasing star formation rates, the $B$-band luminosity evolution of $\mathrm{Sa}$ and $\mathrm{Sb}$ models has a more pronounced maximum at early times whereafter it shows a stronger fading than the Sc model. As well, the $B-V$ colors of $\mathrm{Sa}$ and $\mathrm{Sb}$ models become redder, almost from the very beginning, than those of the Sc model shown here. This is due to the larger fraction of stars formed at early times.

The constant star formation Sd model has almost constant $B$-luminosity from $\leq 2$ to $\geq 12$ Gyr but still shows some reddening, albeit weaker than the Sc model, due to the accumulation of low mass stars.

Note that the gas content drops at very different rates in different types of models, very fast in Sa with $(G / M)_{12 \text { Gyr }}=0.07$ and pretty slowly in Sd models with $(G / M)_{12} \mathrm{Gyr}=0.65$. These global gas contents are also in broad agreement with typical gas-to-total mass ratios of field galaxies if the HI beyond their optical radius is taken into account. Without significant accretion from outside these evolving gas masses set upper limits to the star formation in bursts. In our definition of the burst strength in terms of consumed gas fraction, a 70\% (strong) burst in an old (9 Gyr) and hence already gas-poor Sa galaxy only increases its stellar mass by $0.8 \%$ while a $70 \%$ burst in a 
Table 1. Comparison of the two different burststrength definitions $b:=\frac{\Delta \mathrm{G}}{\mathrm{G}\left(\mathrm{t}_{\text {burst }}\right)}$ and $b_{*}:=\frac{\Delta \mathrm{S}}{\mathrm{S}\left(\mathrm{t}_{0}\right)}$ for galaxies bursting at 6 Gyr.

\begin{tabular}{ccc}
\hline \hline Hubble Type & $b$ & $b_{*}$ \\
\hline Sa & $30 \%$ & $6 \%$ \\
& $70 \%$ & $16 \%$ \\
$\mathrm{Sb}$ & $30 \%$ & $13 \%$ \\
& $70 \%$ & $27 \%$ \\
$\mathrm{Sc}$ & $30 \%$ & $29 \%$ \\
& $70 \%$ & $52 \%$ \\
\hline
\end{tabular}

much more gas-rich 3 Gyr young Sc model would increase its stellar mass by almost a factor of 2 .

Table 1 compares the different definitions of the burst strength parameters for bursts after 6 Gyr of undisturbed evolution.

\section{Comparison with S0 galaxy data}

As a first application of our models we study the origin of the S0 galaxy population in cluster environments. The conventional model of S0 galaxy evolution is a high SFR at the beginning followed by a strong decrease of star formation activity (cf. Sandage 1986; Bruzual \& Charlot 1993; Guiderdoni \& Rocca-Volmerange 1987). In this picture, S0 galaxies are old and have evolved passively most of the time. Recent observations, however, show that the fraction of S0 galaxies in clusters evolves strongly with redshift. We assume that interactions among infalling galaxies and with the cluster environment trigger the evolution. We take the turn-over $M_{B}$ of the Gauss-fit determined by Sandage et al. (1985) to the S0 galaxy luminosity function in Virgo (adjusted to our choice of $H_{0}=65$ ) as our reference for the local average S0 luminosity $\left\langle M_{B}\right\rangle$. Note that the completeness limit for this sample occurs about 5 mag fainter than $\left\langle M_{B}\right\rangle$. The average colors of local $\mathrm{S} 0$ galaxies over the same luminosity range are taken from Buta et al. (1994) and Bower et al. (1992). Table 2 shows the data we use for the local S0 galaxy population, in our comparison with models of interacting and disturbed spiral galaxies described above.

Table 2. Average S0 galaxy data with $1 \sigma$ dispersions.

\begin{tabular}{ccc}
\hline \hline$\left\langle M_{B}\right\rangle$ & $-18.55 \pm 1.5 \mathrm{mag}$ & Sandage et al. (1985) \\
$B-V$ & $0.90 \pm 0.060 \mathrm{mag}$ & Buta et al. (1994) \\
$U-B$ & $0.46 \pm 0.097 \mathrm{mag}$ & Buta et al. (1994) \\
$V-K$ & $3.15 \pm 0.100 \mathrm{mag}$ & Bower et al. (1992) \\
\hline
\end{tabular}

The aim is to find out which effects on the star formation rates in which types of galaxies and at what times do produce colors and luminosities by today in agreement with S0 galaxy data. This kind of investigation is meant to complement dynamical models for the morphological transformation of galaxies in cluster environments (Moore et al. 1996, 1998).

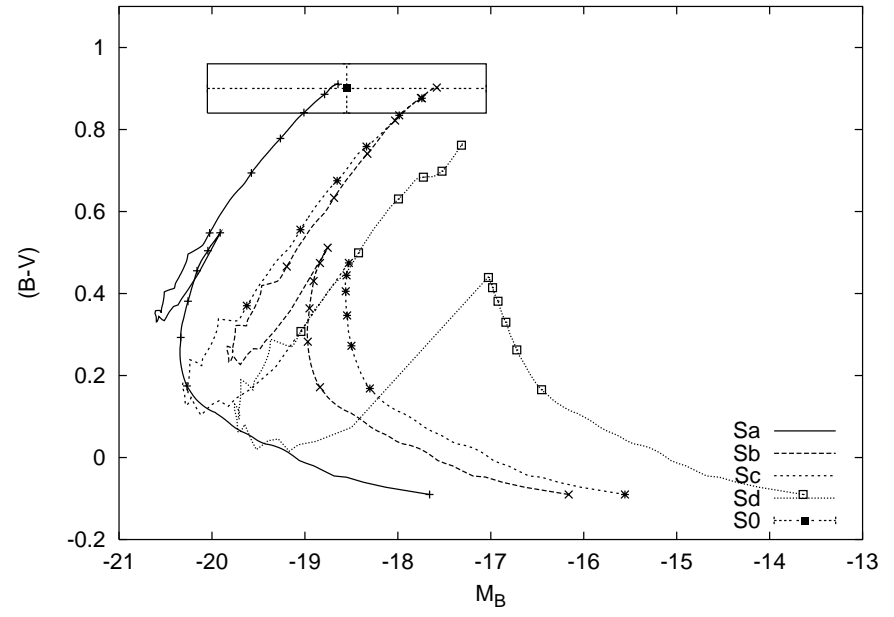

Fig. 3. Color-magnitude diagram for the evolution of spiral galaxies with strong bursts starting at an age of 6 Gyr. The symbols mark timesteps of 1 Gyr.

It was seen from the models alone (Sect. 3) that while in terms of $B-V$ colors at $\sim 12$ Gyr several very different models looked identical, they considerably split up in terms of $B$-band luminosity. We therefore chose to present here our comparison of models and S0 data in terms of color-magnitude diagrams.

\subsection{Starburst models}

In Fig. 3 we show the evolution of model galaxies of types $\mathrm{Sa}, \mathrm{Sb}, \mathrm{Sc}$, and Sd with strong starbursts starting at an age of 6 Gyr in a color-magnitude diagram $(B-V)$ vs. $M_{B}$. The evolutionary tracks start in the lower part of the diagram. Timesteps of 1 Gyr are shown by the symbols on each track. As shown in Figs. 1 and 2 for undisturbed Sc galaxies, all models get brighter and redder, and this evolution is fastest in the earliest stages and gets slower later on. At an age of $6 \mathrm{Gyr}$ all models are assumed to have strong starbursts. Hence, the model galaxies get bluer and brighter very fast and then, with falling SFR, they get redder and fainter again. The strengths of the changes in color and luminosity during the burst depend on galaxy type. The Sd model, with a lot of available gas, passes through a big loop in the color-magnitude diagram, while the Sa model, with much less gas, only shows a small loop. Remember, all models have a burst of the same strength in our definition, i.e. they transform $70 \%$ of their available gas mass into new stars.

The box in the upper left of the color-magnitude diagram indicates the $1 \sigma$ range around the average luminosity and color of the local S0 galaxy population (see also Table 2). The endpoints of the $\mathrm{Sa}, \mathrm{Sb}$, and Sc evolutionary tracks, at an age of $12 \mathrm{Gyr}$, lie within the observed $1 \sigma$ range of local S0 galaxies. Only the Sd model is too blue to reach to within $1 \sigma$ of the photometric properties of S0s.

The following figures only show the end points of galaxy evolution at 12 Gyr. In all plots the sequence of undisturbed galaxies is shown for reference. Lines connect 

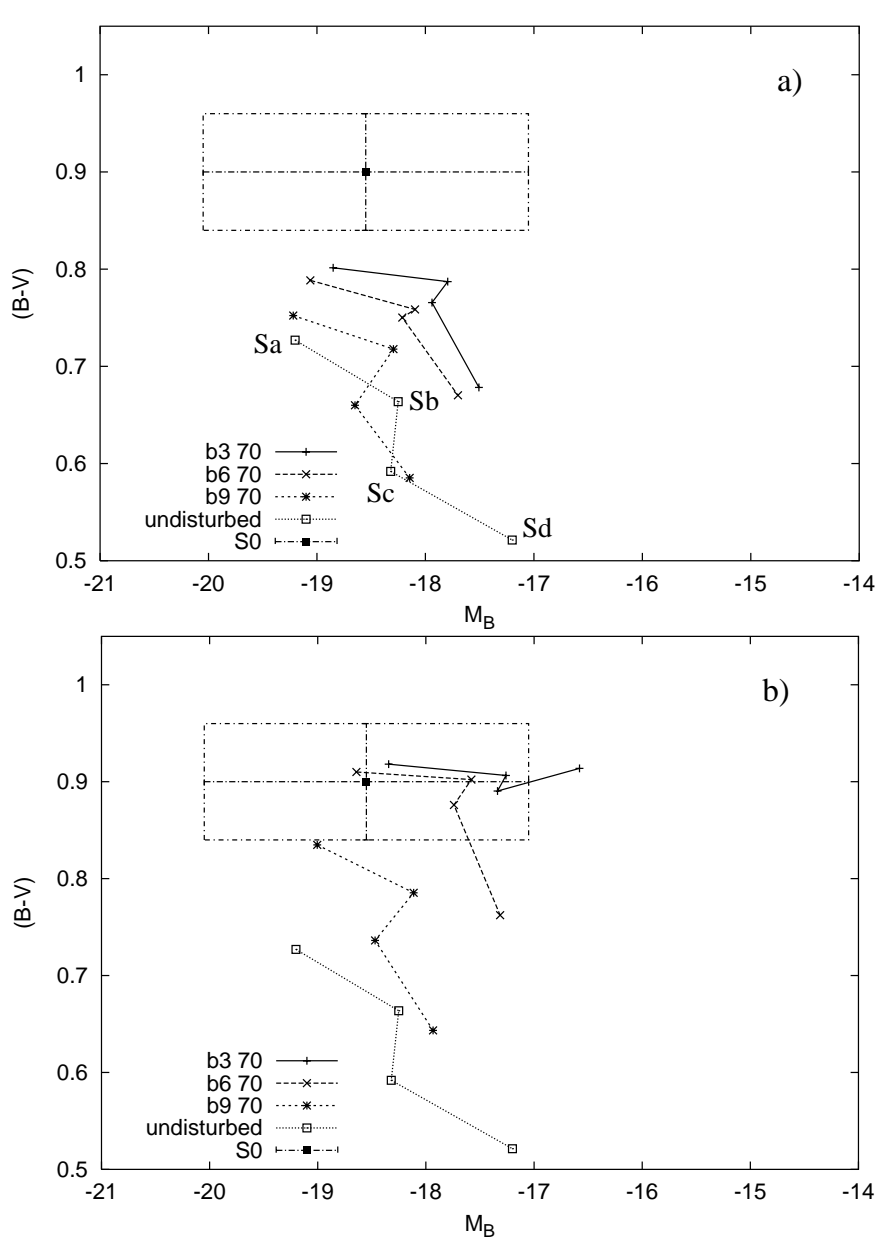

Fig. 4. Difference between burst models with and without residual SFR $\psi_{f}=1.5 M_{\odot} \mathrm{yr}^{-1}$ a) and $\psi_{\mathrm{f}}=0 \mathbf{b}$ ).

the various galaxy types with the same interaction history (from left to right: $\mathrm{Sa}, \mathrm{Sb}, \mathrm{Sc}$, and $\mathrm{Sd}$ ). The box in the upper left again shows the $1 \sigma$ range of the local S0 population.

Figures $4 \mathrm{a}$ and $\mathrm{b}$ show for starburst models the effect of a residual ongoing $\operatorname{SFR}\left(\psi_{\mathrm{f}}=1.5 M_{\odot} \mathrm{yr}^{-1}\right)$ after the burst (Fig. 4a) as compared to starburst models with no ongoing star formation (4b). In both figures strong (70\%) burst at three different times of evolution (3, 6, 9 Gyr) are plotted. The differences are very clear. No model with ongoing low level star formation reaches the luminosity and color range $(1 \sigma)$ of local $\mathrm{S} 0$ galaxies. All model galaxies are too blue. This is the consequence of the ongoing star formation, as the comparison with Fig. $4 \mathrm{~b}$ shows. Without ongoing star formation after the burst several model galaxies reach the $1 \sigma$ range of local $\mathrm{S} 0$ galaxies in luminosity and color. Only the galaxies which have their burst at an evolutionary age of 9 Gyr (this means only 3 Gyr ago) are (still) too blue. The Sa galaxy, however, even after a burst only 3 Gyr ago is already very close to the colors of $\mathrm{S} 0$ galaxies.

We conclude that all spiral galaxies with bursts at ages of 3 or 6 Gyr, except for the Sds, reach both the luminosity and color ranges of S0s by today, and this is not only

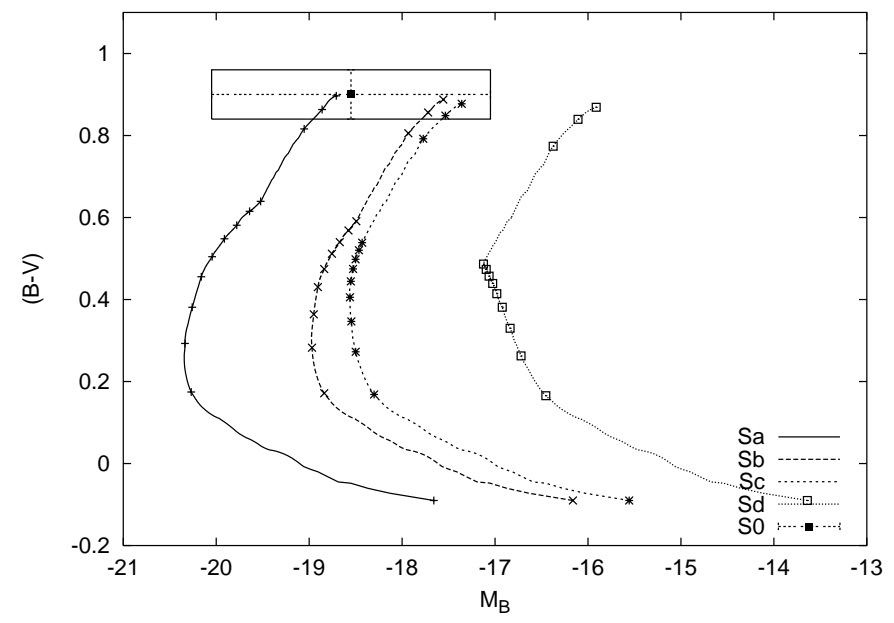

Fig. 5. Color-magnitude diagram for the evolution of spiral galaxies with star formation truncation after 9 Gyr of undisturbed evolution. The symbols mark timesteps of 1 Gyr.

true for $M_{B}$ and $(B-V)$ displayed here but for all other colors available. The Sd galaxies are either too faint (burst at $3 \mathrm{Gyr}$ ) or too blue (bursts at 6 or $9 \mathrm{Gyr}$ ) to resemble by today the average photometric properties of S0 galaxies. An essential prerequisite for post-starburst models to reach the red colors of $\mathrm{S} 0$ galaxies is a complete extinction of SF.

\subsection{Star formation truncation models}

Another interaction scenario is shown in Fig. 5. Here the SFR is truncated after 9 Gyr of undisturbed evolution. It is a color-magnitude diagram like Fig. 3 with the same scale. So the models follow the tracks from the bottom to the top, ending at 12 Gyr with symbols again marking timesteps of 1 Gyr. Like in Fig. 3, the Sa, Sb and Sc models - now with SF truncation - reach the observed range of local S0 galaxies in the upper left, while the Sd model again misses the S0 region. In this scenario, however, the Sd model fails because its luminosity is too low while its color now is red enough, in contrast to the burst scenario. It is also seen that the effect of the SF truncation (the break in the curve) is smaller for the Sa model and gets stronger towards the Sd model. The explanation is that the SFR in an Sa galaxy is already low at this time of evolution and the galaxy is dominated by old stars and rather red already, so a truncation of the SFR does not affect the galaxy too much. The Sd galaxy, on the other hand, is actively forming stars even at an age of 9 Gyr. A truncation of its SFR causes a sudden decrease in the number of young hot stars, and the effect on the galaxy in terms of $B$-band luminosity and color is stronger.

The next two Figures, Figs. 6a and b show SF truncation scenarios, occurring at 3,6, and 9 Gyr of evolution in different spiral galaxy types. In Fig. 6a $B-V$, and in 6b $V-K$ colors are plotted against $M_{B}$. Other colors like $U-B$ or $U-V$ do not give any additional information and therefore are not showen. If the SFR is truncated at 

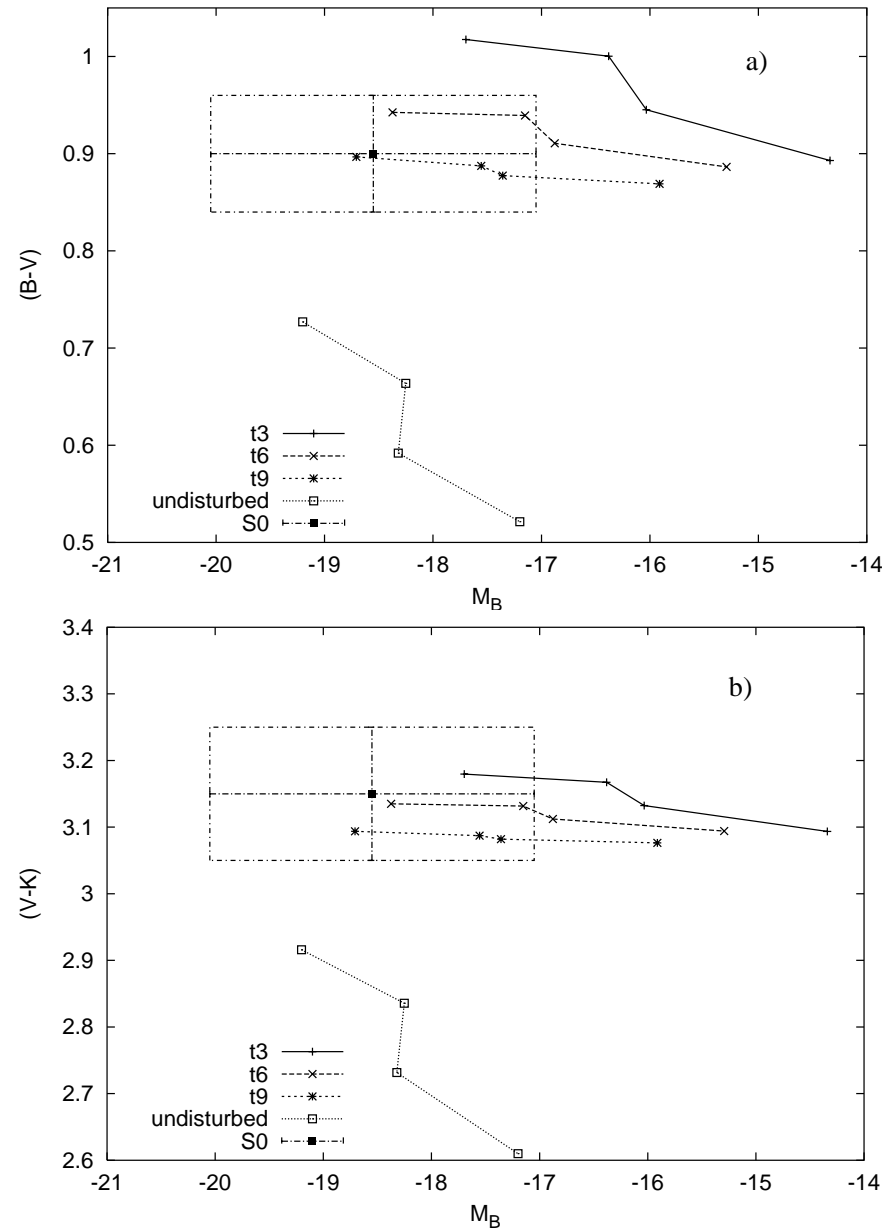

Fig. 6. Effect on galaxy colors and luminosities at $\sim 12$ Gyr of SF truncation in various spiral types (connected by lines to guide the eye) occurring at various evolutionary ages. The two figures show different colors $(B-V) \mathbf{a})$, and $(V-K) \mathbf{b})$.

early times (3 Gyr) the colors become too red ( $\mathrm{Sa}, \mathrm{Sb}$ ) and the luminosities to faint ( $\mathrm{Sb}, \mathrm{Sc}, \mathrm{Sd}$ ) by today. Is the SFR truncated at 6 or $9 \mathrm{Gyr}$, most models reach the $1 \sigma$ range of local S0 galaxies. Only Sd galaxies are an exception, they are too faint because they did not build enough stars to get as bright as an average S0 galaxy. In Fig. 6b the conclusions for models which have a SF truncation after 6 or 9 Gyr are equal to those described above. But in comparison to a) the Sa galaxy is not too red in $V-K$ in case of SF truncation at 3 Gyr. This is due to the fact that the old stars are dominating $V-K$ while in $U-B$ or $B-V$ young hot stars dominate. So the truncation of the SFR is less visible in $V-K$ as compared to $U-B$ or $B-V$.

Hence, the $(B-V)$ vs. $M_{B}$ diagram already contained all the information. The inclusion of $U$ - and $K$-bands does not lead to any further restriction of the manifold of S0 galaxy progenitor models. We conclude that Sa models with SF truncation after 6 or 9 Gyr of evolution fall well into the color and luminosity range of present-day S0 galaxies. $\mathrm{Sb}$ and $\mathrm{Sc}$ galaxies with $\mathrm{SF}$ truncation at 6 or 9 Gyr and at 9 Gyr, respectively, may be the progenitor

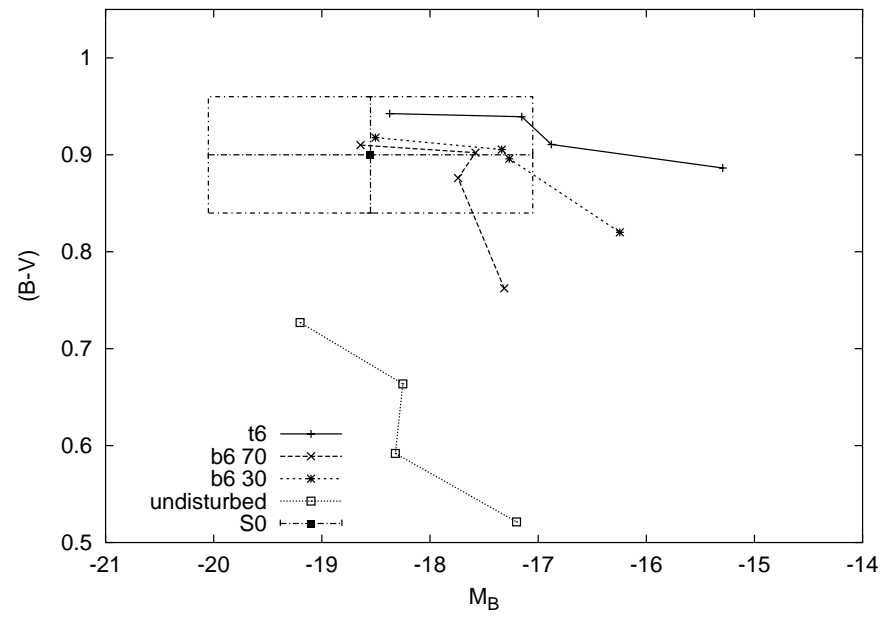

Fig. 7. Comparison of models with various burst strengths, $b=0.7$ and $b=0.3\left(\psi_{\mathrm{f}}=0\right)$ and with SF truncation, all occurring at an age of $6 \mathrm{Gyr}$.

of lower than average luminosity S0s. Late-type Sd spirals with SF truncation at any time are too faint.

\subsection{Effects of the burst strength}

In Fig. 7 we compare different burst strengths and SF truncation (which can also be understood as a $b=0$ model) on galaxies which have their SFR disturbed at 6 Gyr. The burst strengths are $70 \%$ and $30 \%$ without ongoing star formation thereafter. Again, the Sd galaxies lie all outside of the S0 range. Their $B-V$ colors and $B$ luminosities are strongly dependent on burst strength. All other spiral galaxy types lie much closer to each other, in particular the differences between the strong and weak bursts are small. All these galaxies reach the $1 \sigma$ range of today's S0s, only the truncated Sc galaxy marginally misses the S0 luminosity range. Hence, our models show that the burst strength is not an important factor in the transformation of a spiral progenitor into an S0 galaxy. The important factor is the SFR which has go to zero by today, either by a direct truncation, or after a burst.

\subsection{Merger-induced starbursts}

Last, we study the merger scenario. As mentioned before, the high velocity dispersions of galaxies in local rich clusters clearly are unfavorable for galaxy mergers. In infalling galaxy groups, still today, and during early stages of cluster formation, however, galaxy merging may play or have played a role. We restrict ourselves to mergers of equal spiral types, and explore the starburst that may be triggered in the course of merging. These types of mergers double the mass - in stars as well as in gas - of our model galaxies, and also double the luminosity of the merger remnants, corresponding to a brightening by $0.75 \mathrm{mag}$. The colors do not change with respect to the simple starburst models discussed above. Figure 8 shows galaxies which merged at 3,6 , and 9 Gyr and experienced a strong starburst at this 


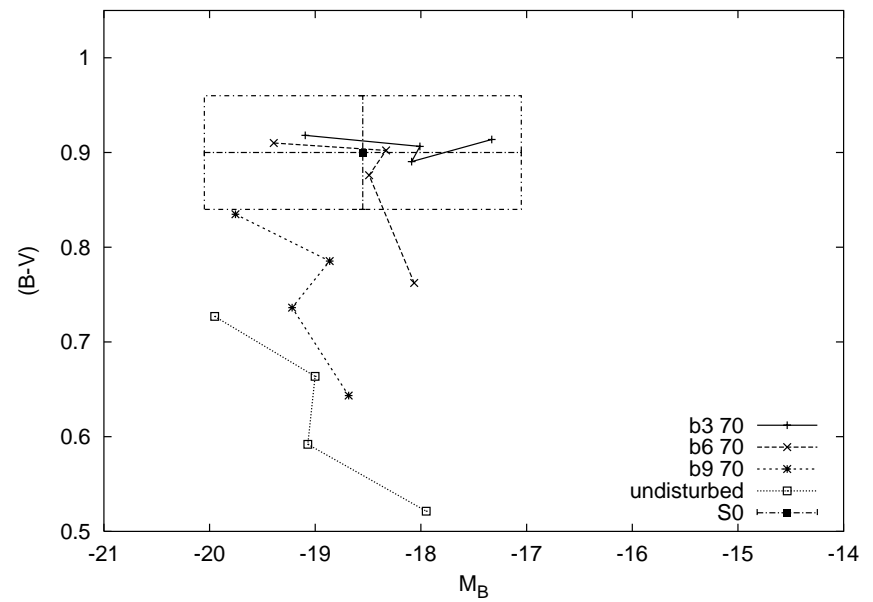

Fig. 8. Color-magnitude diagram for Spiral-Spiral merger models with strong starbursts and SFR $=0$ after the burst

occasion. Comparison with Fig. 4b for starbursts in single galaxies shows this difference of $0.75 \mathrm{mag}$ in luminosity. So, like all others, the Sd-Sd merger remnants are brighter and in the case of a burst at 3 Gyr they now can reach the faint part of the S0 luminosity distribution. The Sa galaxies are pushed by mergers into the luminosity range of bright S0s, while in the non-merger starburst scenario they only reached the average S0 luminosity. Mergers of different normal galaxy types and mergers between a normal and a dwarf galaxy never get brighter than an Sa-Sa merger. Hence, the only ways to produce brighter than average $\mathrm{S} 0$ s are either early-type spiral merger with starbursts induced at 3 or 6 Gyr, or multiple mergers. Sb-Sb and Sc-Sc mergers at 3 or 6 Gyr can well account for the average luminosity S0 population and even early mergers (at 3 Gyr) of late-type Sd galaxies do reach the observed color and luminosity range of present-day S0s. SFRs have to go to zero after the bursts in all cases to obtain red enough colors. Sd - Sd mergers at 6 Gyr and all types of late mergers (at $t \geq 9 \mathrm{Gyr}$ ) remain too blue.

\subsection{Discussion}

Note that we show the $1 \sigma$ range of the local S0 galaxy population in all of our plots and so our models also describes average luminosity progenitor galaxies. Hence, some of the models marginally missing the local S0 $1 \sigma$ box in therms of luminosity may still describe valid ways to transform spirals into S0s. Within infalling groups, it is, of course, also possible that more than two galaxies may merge during their infall into a cluster, giving rise to a bright S0 galaxy.

The results of our photometric study are in good agreement e.g. with Jones et al. (2000) who also quote star formation truncation as the dominant transformation process from field spirals to cluster S0s from analysis of spectral features and HST morphologies of S0 galaxies in clusters at redshift $0.3-0.6$. Our conclusions confirm their results obtained for the brighter part of the S0 population accessible to spectral analyses and goes beyond that study in the inclusion of the fainter part of the local S0 population. On the other hand, more than $40 \%$ of the S0 galaxies in the Coma cluster are found to show evidence for star formation in their central regions during the last $\sim 5 \mathrm{Gyr}$ by Poggianti et al. (2001b) from spectroscopic analyses, in particular at fainter luminosities, pointing towards a starburst prior the star formation truncation, complementing our findings that most pure star formation truncation models have remnants fainter than average S0 galaxies.

Poggianti et al. (2001a) find that the fraction of present-day luminos galaxies that had significant SF between $z=2$ and $z=0.35$ is higher than for lower luminosity galaxies. This as well, is in agreement with our results that luminous S0s must have had starbursts earlier in their evolution while lower luminous ones may also result from pure SF truncation.

We conclude that two scenarious may be responsible for the transformation of field spiral galaxies colors and luminosities into those of S0s. Starbursts and possibly even spiral-spiral mergers seem necessary for the luminous part of the cluster S0 population while SF truncation without any significant preceding enhancement may explain the lower luminous S0s.

Our finding that $\mathrm{S} 0$ galaxies in clusters may result from starbursts in spirals at all ages below about 9 Gyr is not surprising in the sense that more recent bursts may still show features of postburst galaxies and hence not be classified as S0s. Our result that SF truncation, on the other hand, if occuring too early, would make galaxies redder than observed for today's S0s, may have to do with the fact that the hot and dense ICM in the centers of galaxy clusters thought to be responsible for sweeping the gas out of the infalling spirals needs time to pile up and may not be efficient in truncating SF before $z \sim 0.5 \ldots 1$.

\section{Conclusions and outlook}

The fraction of S0 galaxies in clusters is observed to significantly depend on the redshift of the cluster. In nearby clusters the fraction of S0 type galaxies is about $40 \%$, whereas in clusters up to $z=0.5$ it decreases by a factor 3 to 4. Various interaction processes have been discussed that might act to transform spirals from the field galaxy population falling into a cluster into passive S0 galaxies. Starbursts may be triggered by shock waves caused in the ISM when a galaxy falls into the hot dense cluster ICM or by mergers within groups of infalling galaxies. Star formation truncation, on the other hand, may occur in galaxies which lose their gas by tidal interaction or by ram pressure stripping when falling into a cluster.

In this paper we study the origin of S0 galaxies in clusters by means of photometric evolutionary synthesis. Our models calculate the time evolution of global luminosities $U, \ldots, K$ and colors, as well as of the stellar and gaseous content of various types of undisturbed spiral galaxies Sa - Sd. These are parameterized by their respective appropriate star formation histories and models are normalized 
to reproduce, after $\sim 12$ Gyr of evolution, the average $B$ band luminosity locally observed for the respective spiral type. We computed a grid of models for spiral galaxies falling into a cluster and having their SFRs affected by starbursts and/or star formation truncation occurring at various evolutionary times. The luminosities and colors of the disturbed galaxies at an age of $\sim 12$ Gyr are compared to the average observed photometric properties $(B$-band luminosity, $(B-V),(U-B)$, and $(V-K)$ colors $)$ of local S0 galaxies. Our aim was to find out which of the disturbed models reach the color and luminosity range of S0 galaxies in the Virgo and Coma clusters and, hence, to constrain the manifold of transformation processes.

We find that many of our disturbed galaxy models do evolve into the color and luminosity range of local S0 galaxies.

- The strongest constraint is that the SFR after the interaction has to go to zero. Models with even low-level ongoing SF after a burst or after SF truncation remain too blue. If before the total SF truncation a burst occurs, even a strong one, or not, is of minor importance.

- All spiral galaxy types Sa - Sc with starbursts that occurred more than 3 Gyr ago reach S0 galaxy colors and luminosities by today. Sd galaxies with early or late bursts are too faint or too blue, respectively.

- Sa models with SF truncation at ages between 6 and 9 Gyr well account for the photometric properties of present-day $\mathrm{S} 0$ s. Sb galaxies with $\mathrm{SF}$ truncation at 6 or 9 Gyr and Sc galaxies with SF truncation at 9 Gyr may account for the lower luminosity part of the S0 population. Sd galaxies with SF truncation at any time are too faint.

- The strength of a starburst is not an important factor for the development of photometric resemblance to S0 galaxies as long as the SFR after the burst declines enough.

- We find that photometric constraints leave two scenarios viable for the transformation of field spirals into cluster S0s: while low luminosity S0s may be the result of simple SF truncation in spirals earlier than Sd, the higher luminosity S0s require starbursts or even mergers prior to SF extinction.

- Our results imply a difference in the cosmological epoch of the transformation of field spirals to cluster S0s in the two scenarios. Starbursts occuring in spirals at all ages lower than about 9 Gyr leave remnants with average $\mathrm{S} 0$ colors while SF truncation should not occur at epochs earlier than $z \sim 0.5, \ldots 1$ in order not to make the remnants redder than present-day S0s. This may be related to the fact that the hot and dense ICM thought to be responsible for sweeping the gas out of the infalling spirals and truncating SF may not yet be efficient at earlier epochs.

Our photometric evolutionary synthesis approach is meant to complement dynamical simulations and studies of spectral features. A more detailed analysis of the redshift evolution of the properties and relative numbers of S0 galaxies in clusters will be the next step in our attempt to constrain possible evolutionary paths from the spiralrich field galaxy population to the S0-rich local cluster population. Spectral modeling including evolutionary and cosmological corrections will have to be compared to observations throughout clusters of various richness, degree of relaxation, and redshift.

Acknowledgements. We thank our referee, Dr. Bianca Poggianti for a very prompt, insightful and constructive report.

\section{References}

Abadi, M. G., Moore, B., \& Bower, R. G. 1999, MNRAS, 308, 947

Bahcall, J. N., Flynn, C., \& Gould, A. 1992, ApJ, 389, 234

Balogh, M. L., Navarro, J. F., \& Morris, S. L. 2000, ApJ, 540, 113

Barnes, J. E., \& Hernquist, L. 1996, ApJ, 471, 115

Biermann, P., \& Tinsley, B. M. 1975, A\&A, 41, 441

Bower, R. G., Lucey, J. R., \& Ellis, R. S. 1992, MNRAS, 254, 589

Bravo-Alfaro, H., Cayatte, V., van Gorkom, J. H., \& Balkowski, C. 2000, AJ, 119,580

Bruzual, A. G., \& Charlot, S. 1993, ApJ, 405, 538

Buta, R., Mitra, S., de Vaucouleurs, G., \& Corwin, H. G. 1994, AJ, 107, 118

Butcher, H., \& Oemler, A. 1978, ApJ, 219, 18

Cayatte, V., van Gorkom, J. H., Balkowski, C., \& Kotanyi, C. 1990, AJ, 100, 604

Couch, W. J., Barger, A. J., Smail, I., Ellis, R. S., \& Sharples, R. M. 1998, ApJ, 497, 188

Dressler, A. 1980, ApJ, 236, 351

Dressler, A., \& Gunn, J. E. 1992, ApJS, 78, 1

Dressler, A., Oemler, A. J., Couch, W. J., et al. 1997, ApJ, 490, 577

Dressler, A., Smail, I., Poggianti, B. M., et al. 1999, ApJS, 122, 51

Fasano, G., Poggianti, B. M., Couch, W. J., et al. 2000, ApJ, 542,673

Fritze-v. Alvensleben, U., \& Gerhard, O. E. 1994, A\&A, 285, 751

Fujita, Y. 2001, ApJ, 550, 612

Gavazzi, G., Contursi, A., Carrasco, L., et al. 1995, A\&A, 304, 325

Guiderdoni, B., \& Rocca-Volmerange, B. 1987, A\&A, 186, 1

Gunn, J. E., \& Gott, J. R. I. 1972, ApJ, 176, 1

Jones, L., Smail, I., \& Couch, W. J. 2000, ApJ, 528, 118

Kenney, J. D. P., \& Koopmann, R. A. 1999, AJ, 117, 181

Kennicutt, R. C. 1998, ApJ, 498, 541

Kodama, T., \& Smail, I. 2001, MNRAS, 326, 637

Kuntschner, H. 2000, MNRAS, 315, 184

Moore, B., Katz, N., Lake, G., Dressler, A., \& Oemler, A. 1996, Nature, 379, 613

Moore, B., Lake, G., \& Katz, N. 1998, ApJ, 495, 139

Poggianti, B. M., Smail, I., Dressler, A., et al. 1999, ApJ, 518, 576

Poggianti, B. M., Bridges, T. J., Mobasher, et al. 2001, ApJ, 562, 689 
Poggianti, B. M., Bridges, T. J., Carter, D., et al. 2001, ApJ, 563, 118

Poggianti, B. M., \& van Gorkom, J. H. 2001, in Gas and Galaxy Evolution, ed. J. E. Hibbard, M. Rupen, \& J. H. van Gorkom, ASP Conf. Proc., 240, 599

Quilis, V., Moore, B., \& Bower, R. 2000, Science, 288, 1617

Rocha-Pinto, H. J., \& Maciel, W. J. 1998, A\&A, 339, 791

Sandage, A. 1986, A\&A, 161, 89

Sandage, A., Binggeli, B., \& Tammann, G. A. 1985, AJ, 90, 1759

Scalo, J. M. 1986, Fund. Cosmic Phys., 11, 1
Schweizer, F. 1999, Ap\&SS, 267, 299

Smail, I., Dressler, A., Couch, W. J., et al. 1997, ApJS, 110, 213

Smail, I., Kuntschner, H., Kodama, T., et al. 2001, MNRAS, 323,839

Tinsley, B. M. 1980, Fund. Cosmic Phys., 5, 287

Toomre, A., \& Toomre, J. 1972, ApJ, 178, 623

Weilbacher, P. M., Duc, P.-A., Fritze-v. Alvensleben, U., Martin, P., \& Fricke, K. J. 2000, A\&A, 358, 819

Ziegler, B. L. 2000, Rev. Mod. Astr., 13, 211 